\title{
Subwavelength Structure of the Evanescent Field of an Optical Bloch Wave
}

\author{
R. J. P. Engelen, ${ }^{1}$ D. Mori, ${ }^{2}$ T. Baba, ${ }^{2}$ and L. Kuipers ${ }^{1}$ \\ ${ }^{1}$ Center for Nanophotonics, FOM Institute for Atomic and Molecular Physics (AMOLF), Kruislaan 407, \\ 1098 SJ Amsterdam, The Netherlands \\ ${ }^{2}$ Yokohama National University, Department of Electrical and Computer Engineering, \\ 79-5 Tokiwadai, Hodogayaku, Yokohama 240-8501, Japan
}

(Received 27 February 2008; revised manuscript received 29 October 2008; published 13 January 2009; corrected 16 January 2009)

\begin{abstract}
The periodic structure of a photonic crystal causes the propagating waves to be governed by Bloch's theorem: they are composed of multiple wave vectors or harmonics. We found, by measuring the field with phase-sensitive near-field microscopy, that the evanescent field of the composite Bloch wave decays nonexponentially as a function of height. Even the individual Bloch harmonics, having only a single wave vector along the propagation direction, do not necessarily decay single exponentially, which has its origin in the spread of wave vectors required to confine the light to the waveguide. The complex decay leads to an evolution of the mode pattern as a function of the height above the structure.
\end{abstract}

DOI: 10.1103/PhysRevLett.102.023902

PACS numbers: 42.70.Qs, 42.82.Et, 68.37.Uv

Sir Isaac Newton was the first to report on the evanescent field that extends approximately "ten hundred thousandth Part of an Inch" (25 nm) above the surface [1]. The evanescent field is exploited for a broad range of applications, ranging from coupling of light in and out of structures [2] to two-dimensional Bose-Einstein condensation [3], strong coupling [4], sensing [5], subwavelength focusing [6], and microscopy [7-9].

The textbook example of an evanescent wave is that of total internal reflection at a planar interface from a high dielectric constant $(\epsilon)$ to a low- $\epsilon$ material with $\epsilon_{\text {low }}$. Above the interface $(z>0)$ the field amplitude decays according to

$$
E(z)=E(0) e^{i z \sqrt{k_{0}^{2}-k_{\|}^{2}}}, \quad \text { with } k_{0}^{2}=\epsilon_{\mathrm{low}} \frac{\omega^{2}}{c^{2}},
$$

where $\omega$ is the optical frequency, $c$ is the speed of light in vacuum, and $k_{\|}$is the wave vector parallel to the interface. For evanescent waves at flat planar interfaces, the exponent is real and negative resulting in an exponentially decaying field away from the interface. The evanescent field above periodically patterned surfaces may, however, be different.

An intriguing class of periodically structured optical materials are photonic crystals, in which materials with a high and a low $\epsilon$ are arranged in a lattice [10,11]. Light in a photonic crystal must obey Bloch's theorem, which dictates that the amplitude of a wave must conform to the imposed periodicity [12]. The resulting wave function $\psi$ in a medium periodic in $y$, can be described as

$$
\begin{aligned}
\psi(x, y, z) & =u_{k}(x, y, z) \exp (i k y), \\
\text { where } u_{k}(x, y, z) & =u_{k}(x, y+a, z) .
\end{aligned}
$$

The above equation, with wave vector $k$ and a periodic amplitude modulation $u_{k}(x, y, z)$, with the same period $(a)$ as the lattice, can be rewritten as a Fourier series of plane waves:

$$
\psi(x, y, z)=\sum_{m} a_{m}(x, z) \exp \left[i\left(k+m \frac{2 \pi}{a}\right) y\right], \text { and } m \in \mathbb{Z} \text {. }
$$

Equation (3) shows that the individual cosinelike plane waves that together make up the Bloch wave, the harmonics, each have an amplitude $a_{m}$. Their wave vector is $k$ plus an integer $(m)$ number of the reciprocal lattice period $2 \pi / a$. Based on Eq. (2), one might naively expect an exponential decay for the evanescent field, governed by the magnitude of $k_{\|}(=k)$ [see Eq. (1)]. The multiple harmonics in Eq. (3), however, suggest that the decay is multiexponential [13].

In this Letter, we have investigated the evanescent field above a two-dimensional photonic crystal waveguide with three-dimensional near-field microscopy. We show both with experiments and calculations that both the Bloch nature of the guided light but also the confinement of light in a narrow waveguide, has a profound effect on the evanescent field above the structure. Also the field patterns above the structure strongly evolve with height.

The photonic crystal structure we investigated, is a 200$\mathrm{nm}$ thick air-bridge membrane of $\mathrm{Si}$, which contains an hexagonal lattice (period $a=456 \mathrm{~nm}$ ) of air holes (radius $140 \mathrm{~nm}$ ). A single row of unperforated holes acts as a waveguide. At each side of the waveguide, 10 rows of holes provide ample confinement of the light by the photonic band gap [14]. The transmission loss due to fabrication disorder is estimated to be a few $\mathrm{dB} / \mathrm{mm}$, which constitutes a negligible loss at the length scales relevant for the work presented here $(<100 \mu \mathrm{m})$. Figure 1 shows a portion of the calculated dispersion relation of the waveguide under investigation. The encircled intersections indicate the wave vectors, each spaced $2 \pi / a$ apart, that together form the Bloch wave, that are excited at $\omega=$ 0.298 (units of $2 \pi c / a$ ). Note that the nonencircled set of intersections corresponds to the backward propagating wave. 


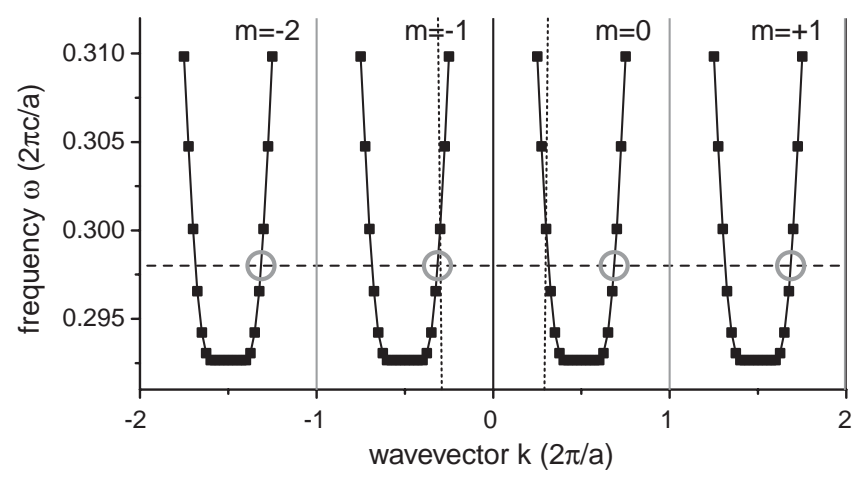

FIG. 1. Portion of the dispersion relation of the photonic crystal waveguide under investigation, obtained via 3D FDTD simulations (solid squares). The solid lines are guides to the eye. An excited Bloch mode at $\omega=0.298$ has multiple wave vectors (encircled intersections), spaced $(2 \pi / a)$ apart. The vacuum mode (or light line, $\omega=c|k|$ ) is depicted with the dotted lines.

We measured the optical field above the structure with a phase-sensitive near-field microscope [15]. Laser light of $\omega=0.298(\lambda=1530 \mathrm{~nm})$ was coupled into the waveguide by end-fire coupling. By scanning a metal-coated tapered optical fiber over the structure, we obtain a map of the optical field above the structure. The scanning setup is incorporated in one branch of an interferometer. The resulting interference signal is proportional to the electric $(E$-)field amplitude in the structure and also contains the phase of the light in the structure. The diameter of the probe aperture $(200 \mathrm{~nm})$ determines, to first approximation, the detection area of $3.1 \times 10^{-14} \mathrm{~m}^{2}$. Figure 2(a) shows the power density of the field, at $\omega=0.298$ for a constant probe-sample distance where the distance is $10 \mathrm{~nm}$. The measurement clearly shows that the light is confined to the waveguide, centered along the line $x=$ $2.6 \mu \mathrm{m}$.

By Fourier transforming the measured complex field along the direction of the waveguide ( $y$ direction), we retrieve the periodic components of the field: the wave vectors $k_{y}$ [16]. Figure 2(b) shows the power spectrum obtained by summing the square of the amplitude of the Fourier transforms for all $x$ values. In agreement with the dispersion relation shown in Fig. 1, we find peaks at $k_{y}=$ $0.68 \pm m$ in normalized units of $2 \pi / a$, with $m$ being an integer. The asterisks in Fig. 2(b) indicate the wave vectors of the wave reflected at the end facet of the waveguide.

In order to recover the spatial distribution of each Bloch harmonic, we applied a Fourier filter to the complex field data underlying Fig. 2(a). Figure 2(c) shows the Fourier filtered data, with a Gaussian selection window centered around $k_{y}=0.68$ and with a width of 0.02 [see Fig. 2(b)]. We define the harmonic with $k_{y}=0.68$ as the fundamental Bloch harmonic with $m=0$. The same filter is applied for the $m=-1$ harmonic $\left(k_{y}=-0.32\right)$, of which the results are depicted in Fig. 2(d). For the fundamental wave vector we find a similar distribution as in Fig. 2(a): the power density is highest in the center of the image, around $x=$
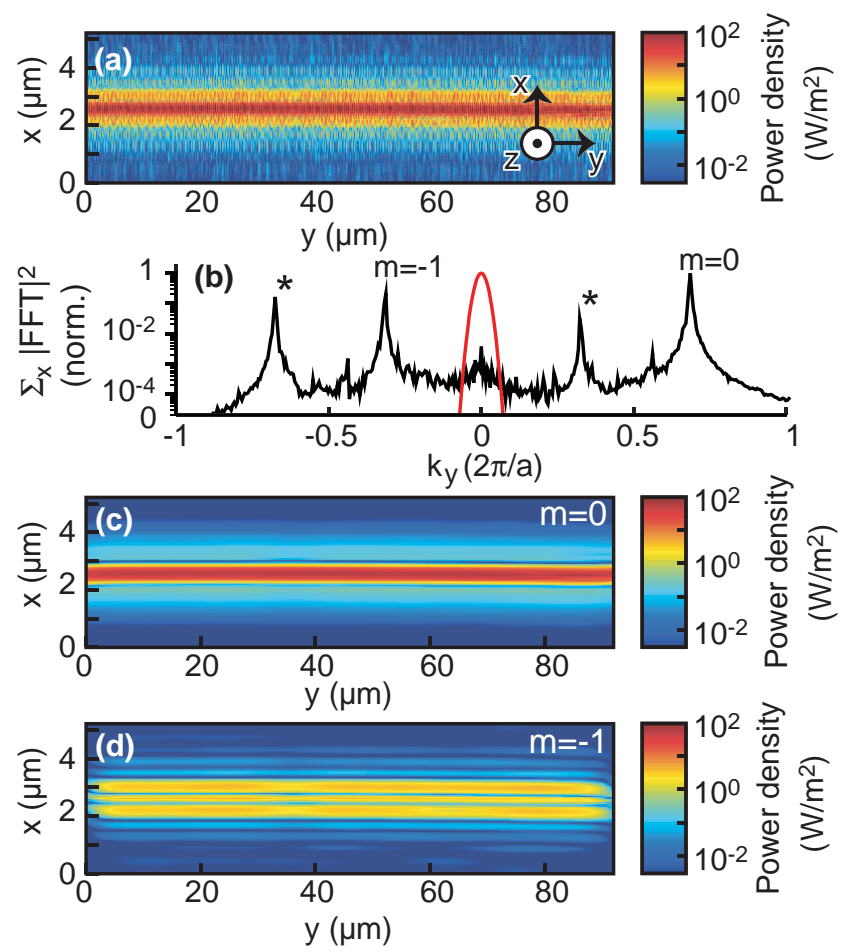

FIG. 2 (color). (a) Color-scale (logarithmic) image of the measured power density distribution of $96 \times 2048$ pixels. The waveguide is centered along the $x=2.6 \mu \mathrm{m}$ line. (b) Power spectrum of the $k_{y}$ wave vectors present in the structure, obtained via Fourier analysis of (a). Two Bloch harmonics are visible ( $m=-1$ and $m=0$ ) and two wave vectors of the reflected Bloch wave (*). The red line depicts the window of the Fourier filter (not necessarily at $k_{y}=0$ ), used to extract the power density distribution of the fundamental and $m=-1$ harmonic, respectively (c),(d), obtained via Fourier filtering the complex phase-sensitive $E$-field distribution underlying (a).

$2.6 \mu \mathrm{m}$. The power density distribution of the $m=-1$ harmonic is, however, more extended into the crystal region. We attribute this difference to the intricate modal pattern of the composite Bloch wave, which exhibits stronger amplitude variations towards the crystal region, requiring the harmonics $m \neq 0$ to be stronger.

To determine the decay of the evanescent field above the photonic crystal waveguide, we performed a series of measurements as a function of the probe-sample distance $z$. We used appropriate Fourier filtering (see above), to recover the power density for the harmonics with $m=$ $-2,-1,0$, and +1 . These harmonics may have a different lateral power distributions [the amplitudes $a_{m}$ in Eq. (3) depend on $x$ ]. We therefore integrated over the distribution perpendicular to the waveguide, to obtain the power per unit length of waveguide. The power as a function of height is depicted in Fig. 3.

Perhaps surprisingly, the curves show that each harmonic decays with a different slope as the height increases, until the curves level off at a power per unit length of $\sim 3 \times 10^{-11} \mathrm{~W} / \mathrm{m}$, where the noise level of the analysis is reached. The harmonics with the largest wave 


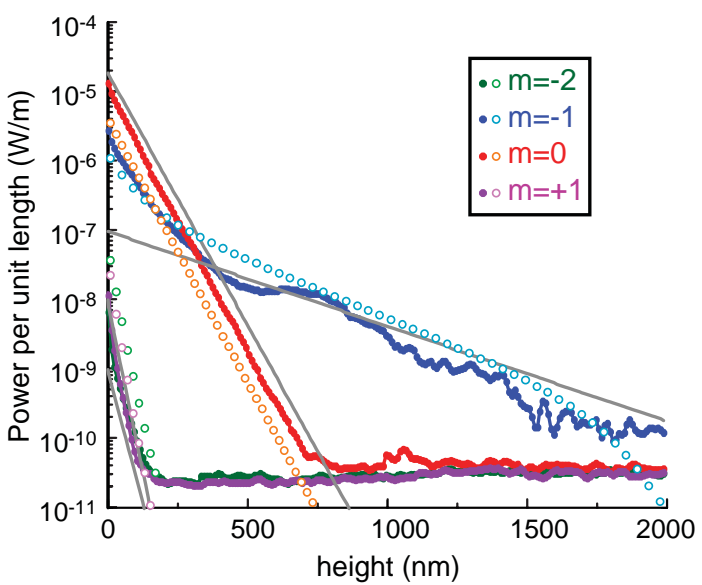

FIG. 3 (color). Measured power in each of the Bloch harmonics as a function of probe-sample height $z$ for four harmonics (see legend). Both the measurement results (solid dots) and the 3 D FDTD results (circles) are shown. The gray lines indicate the decay expected based on Eq. (1) and are offset for clarity.

vectors $(m=-2$ and $m=+1)$, have the strongest decay $\left(\approx 20 \mu \mathrm{m}^{-1}\right)$, whereas the harmonic with the smallest wave vector $(m=-1)$, decays very slowly with height (decay of $\approx 2 \mu \mathrm{m}^{-1}$ ). The ensemble of these harmonics together form the Bloch wave, while the individual harmonics are not a solution to Maxwell's equations. Obviously, the Bloch wave itself, which is composed of these harmonics, therefore does not exhibit a singleexponential decay.

The differences in decay can be understood by considering Eq. (1), since each harmonic has a different wave vector $k_{\|}$, the decay must consequently also differ. Since the parallel wave vectors of the $m=-2$ and $m=+1$ harmonic are much larger than the low- $\epsilon$ wave vector (air), their decay is rapid. The decays expected based on Eq. (1) are depicted with the solid lines, but they do not fully describe the measured decays. In particular, a strong deviation is found for the $m=-1$ harmonic: between 0 and $400 \mathrm{~nm}$ : the experimentally found decay is much stronger than a single-exponential decay expected based on Eq. (1).

We have performed 3D finite-difference time-domain (FDTD) simulations [17] to validate our experimental results and to confirm the deviation from a singleexponential decay. We applied a similar Fourier analysis to the FDTD data to resolve the individual Bloch harmonics. The results are also depicted in Fig. 3. We concluded from the comparison of the results that there is qualitative agreement between FDTD results and experiment. There are some differences in relative amplitude between the harmonics, which we attribute to the wave vectordependent coupling to the near-field probe [18].

Key to the full understanding of the complex decay of the Bloch harmonics and the composite Bloch wave are the lateral modal profiles (perpendicular to the waveguide direction) as a function of height. We have plotted the experimental and simulated results for the fundamental harmonic in Figs. 4(a) and 4(b), respectively, which clearly are in good agreement. The evanescent field has nodes [arrows in Fig. 4(b)], with fields with opposite signs on either side, as is indicated by "+" and "-". On close examination, one can observe that the field profile broadens as the height is increased: the nodes in Figs. 4(a) and 4(b) shift outward as the mode pattern changes as a function of height. This is quite in contrast to what one would naively expect based on Eqs. (1)-(3) as these equations predict only an amplitude decrease, while the mode pattern does not change as a function of height.
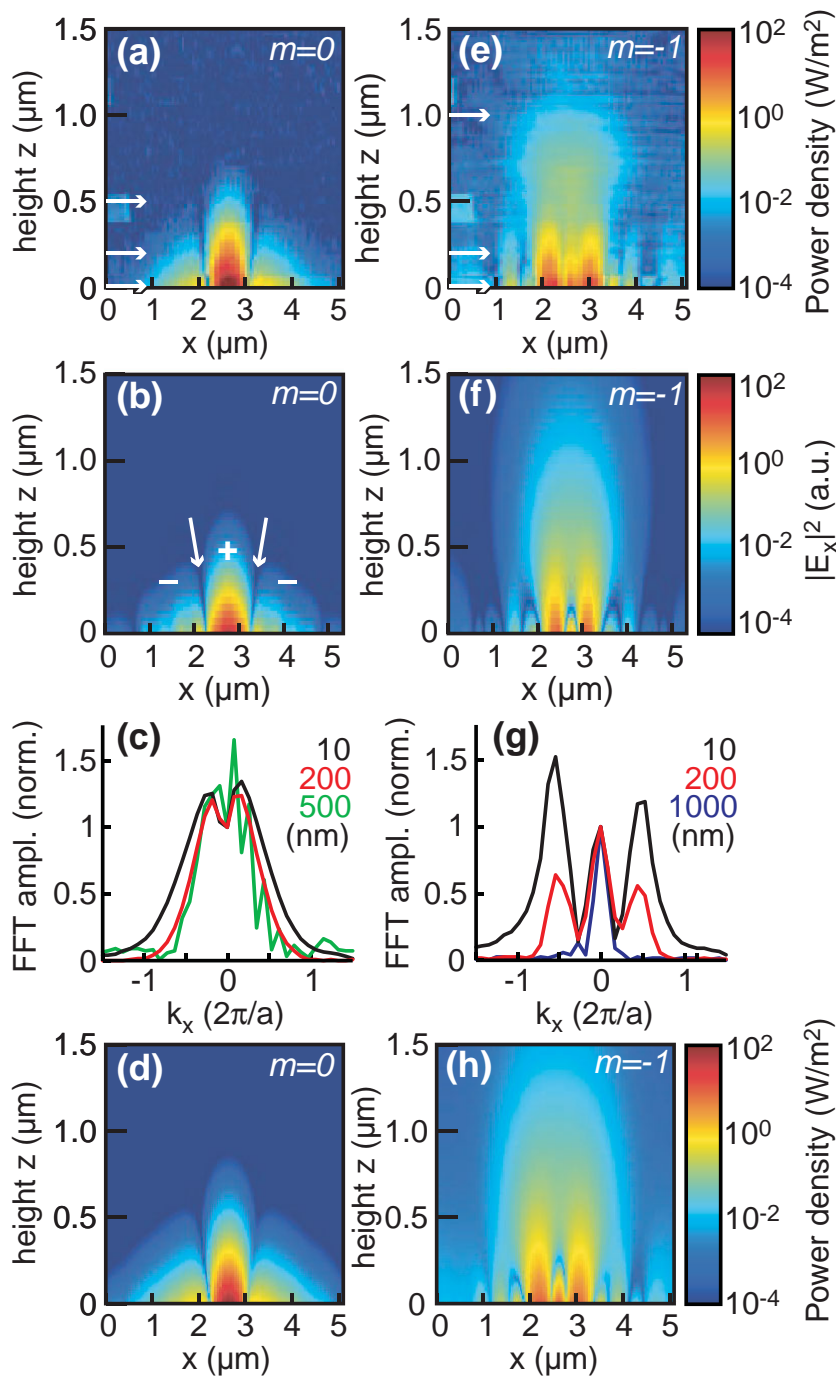

FIG. 4 (color). (a),(b) Power density distribution of the fundamental harmonic as a function of height, measured (a) and from FDTD simulation (b). (c) Amplitude of $k_{x}$ wave vectors obtained via Fourier transformation of the lateral field at heights indicated by the arrows in (a), normalized to the amplitude at $k_{x}=0$. (d) Calculated power density distribution by including the decay of $k_{x}$ wave vectors [see Eq. (4)], using the measurements at $10 \mathrm{~nm}$ as a starting point. (e)-(h) Same as (a)-(d) for the $m=$ -1 Bloch harmonic. 
We observe even stronger pattern changes in the evanescent field of the $m=-1$ harmonic [see Figs. 4(e) and 4(f)], for both the experimental result and the FDTD simulation. In Fig. 4(f), several nodes are visible at a height of $200 \mathrm{~nm}$. As the height is increased, only a smooth profile without nodes remains at a height of $1000 \mathrm{~nm}$.

Both the observation of the changes in modal pattern in the images in Figs. 4 as well as the non-single-exponential decay of the $m=-1$ harmonic seen in Fig. 3 deviate from the single-exponential decay suggested by a naive interpretation of Eq. (1). The explanation for the deviations from this simple behavior lies in the lateral fields of the harmonics. To build up these complex modal patterns, a range of $k_{x}$ wave vectors is required. If the magnitude of the $k_{x}$ wave vectors is comparable to or larger than that of $k_{y}$, they need to be taken into account when calculating the decay or the modal pattern of the evanescent field. The $k_{x}$ wave vectors that make up the lateral patterns are calculated by Fourier transforming the fields and are depicted in Figs. 4(c) and 4(g) for the fundamental and $m=-1$ harmonic, respectively. In both figures, the higher $k_{x}$ values vanish most rapidly as the height increases. Because of the broad range of $k_{x}$ wave vectors, even a single Bloch harmonic does not decay single exponentially.

By substituting $\sqrt{k_{x}^{2}+k_{y}^{2}}$ for $k_{\|}$in Eq. (1) we can reconstruct the evanescent field by inverse Fourier transformation $\left(\mathcal{F}^{-1}\right)$ :

$$
E(x, y, z)=\mathcal{F}^{-1}\left\{\tilde{E}\left(k_{x}, k_{y}, z=0\right) e^{i z \sqrt{k_{0}^{2}-k_{x}^{2}-k_{y}^{2}}}\right\} .
$$

In this equation, the $E$-field amplitude of each wave vector component $\tilde{E}\left(k_{x}, k_{y}, z\right)$ is calculated using only the wave vectors at $z=0$. We assume that all wave vector components are evanescent (i.e., $k_{x}^{2}+k_{y}^{2}>k_{0}^{2}$ ). Since a broad range of $k_{x}$ wave vectors are present in the field of the light in the waveguide, the decay is more complex than a single exponential. We demonstrated the validity of the above argumentation by calculating the evanescent field pattern of each harmonic, using only the experimental $E$-field data at $z=10 \mathrm{~nm}(z \approx 0)$ as a starting point. The result is presented in Figs. 4(d) and 4(h), which clearly show excellent agreement with both the full 3D experimental data [Figs. 4(a) and 4(e)] as well as with the 3D FDTD simulation data [Figs. 4(b) and 4(f)].

In conclusion, we have investigated the decay and pattern changes of the evanescent field above a photonic crystal waveguide. Two effects were found that influence the subwavelength pattern and decay of the field. First, the Bloch nature of the propagating mode is build from an ensemble of wave vectors $\left(k_{y}\right)$, each with their own decay constants. Second, the strong confinement of the light in the narrow waveguide requires a broad range of wave vectors $\left(k_{x}\right)$ perpendicular to the direction of propagation. The influence of the wave vectors in the $x$ direction results in strong changes of the modal pattern as a function of height above the photonic crystal waveguide, which were found in both near-field experiments and 3D FDTD simulations.

Both the measurements and the simulations show that nanostructured optical materials can have rich evanescent field patterns. This property may be exploited by engineering the geometry such that a specific (subwavelength) evanescent field pattern is obtained, by tuning the $k_{x}$ wave vectors. This may, for example, be exploited in optical trapping or manipulation of nanoparticles or Bose-Einstein condensates. Promising results have already been demonstrated for tuning of the $k_{y}$ wave vectors in socalled dispersion engineering in photonic crystal waveguides $[19,20]$.

The authors acknowledge Femius Koenderink and Silvia Vignolini for enlightening discussions. This work is part of the research program of FOM, which is financially supported by the NWO. Support of the EC-funded project PhOREMOST is gratefully acknowledged. This work is also partially supported by NanoNed, a nanotechnology program of the Dutch Ministry of Economic affairs.

[1] I. Newton, Opticks (William Innys, London, 1730), Book III, Qu. 29, 4th ed., p. 346.

[2] M. Cai, O. Painter, and K. J. Vahala, Phys. Rev. Lett. 85, 74 (2000).

[3] D. Rychtarik et al., Phys. Rev. Lett. 92, 173003 (2004).

[4] T. Aoki et al., Nature (London) 443, 671 (2006).

[5] Y. Raichlin, A. Millo, and A. Katzir, Phys. Rev. Lett. 93, 185703 (2004).

[6] R. Merlin, Science 317, 927 (2007).

[7] M.F. Crommie, C.P. Lutz, and D.M. Eigler, Nature (London) 363, 524 (1993).

[8] L. Kastrup et al., Phys. Rev. Lett. 94, 178104 (2005).

[9] D. M. Profunser, O. B. Wright, and O. Matsuda, Phys. Rev. Lett. 97, 055502 (2006).

[10] See, for example, Photonic Crystals and Light Localization in the 21st Century, edited by C.M. Soukoulis (Kluwer Academic, Dordrecht, The Netherlands, 2001).

[11] S. Noda et al., Science 289, 604 (2000).

[12] P. St. J. Russell, Appl. Phys. B 39, 231 (1986).

[13] S. I. Bozhevolnyi et al., Phys. Rev. B 66, 235204 (2002).

[14] Z. Y. Li and K. M. Ho, Phys. Rev. Lett. 92, 063904 (2004).

[15] M. L. M. Balistreri et al., Science 294, 1080 (2001).

[16] H. Gersen et al., Phys. Rev. Lett. 94, 123901 (2005).

[17] C. T. Chan, Q. L. Yu, and K. M. Ho, Phys. Rev. B 51, 16635 (1995). A unit cell of $23 \times 267 \times 210$ pixels $(x \times$ $y \times z$ ) was used, corresponding to $1 \times 11.6 \times 9.1$ lattice periods, with absorbing boundaries conditions for $y$ and $z$ and periodic boundaries for $x$.

[18] I. P. Radko, S. I. Bozhevolnyi, and N. Gregersen, Appl. Opt. 45, 4054 (2006).

[19] L. H. Frandsen et al., Opt. Express 14, 9444 (2006).

[20] M. D. Settle et al., Opt. Express 15, 219 (2007). 